\title{
Determination of sialic acids in infant formula by chromatographic methods: A comparison of high-performance anion-exchange chromatography with pulsed amperometric detection and ultra-high-performance liquid chromatography methods
}

\author{
D. C. Hurum ${ }^{1}$ and J. S. Rohrer \\ Dionex Products, Thermo Fisher Scientific, Sunnyvale, CA 94085
}

\begin{abstract}
Sialic acid determination in an infant formula presents many challenges, including efficient sialic acid release from glycoconjugates, effective sample preparation, and rugged chromatography. This work compares 2 chromatographic assays developed for determination of sialic acids in infant formula. Prior to chromatography, both assays release sialic acids by acid hydrolysis and treat the hydrolysate with a subsequent anionexchange sample preparation. Both high-performance anion-exchange chromatography with pulsed amperometric detection (HPAEC-PAD) and fluorescence ultra-high-performance liquid chromatography (UHPLC) sample analysis methods were evaluated to compare assay performance and convenience. Calibration ranges were chosen to encompass the expected amounts of 2 sialic acids in infant formula: $\mathrm{N}$-acetylneuraminic acid (Neu5Ac) and $N$-glycolylneuraminic acid (Neu5Gc). Response was linear by either method with coefficients of determination of 1.00 by HPAEC-PAD between 5.0 and 100 pmol of Neu5Ac and between 0.34 and 6.8 pmol of Neu5Gc and $>0.99$ by UHPLC between 5.0 and $260 \mathrm{pmol}$ of Neu5Ac and between 0.20 and $9.8 \mathrm{pmol}$ of Neu5Gc. Both methods had sufficient sensitivity to determine these sialic acids in infant formula. Three infant formulas were analyzed to evaluate accuracy and precision of the assays. The HPAEC-PAD assay was found to be faster overall and the UHPLC assay was more sensitive. Reaction efficiency, and therefore sensitivity, was dependent on the sample matrix. This work illustrates sample-specific complexity that must be considered in choosing an assay.
\end{abstract}

Key words: infant formula, sialic acid, high-performance anion-exchange chromatography with pulsed amperometric detection, ultra-high-performance liquid chromatography

Received September 27, 2011.

Accepted November 17, 2011.

${ }^{1}$ Corresponding author: Deanna.Hurum@thermofisher.com

\section{INTRODUCTION}

Dietary sialic acids are thought to play important roles in infant development, having been shown to affect both immune system and cognitive development (Wang and Brand-Miller, 2003). Many neuraminic acids have been identified in human milk; however, $\mathrm{N}$ acetylneuraminic acid (Neu5Ac) is predominant and $\mathrm{N}$-glycolylneuraminic acid (Neu5Gc) is usually absent. In comparison, bovine milk contains approximately $5 \%$ Neu5Gc (Wang and Brand-Miller, 2003) and has been shown to contain less than $25 \%$ of the total sialic acid content of human milk (Wang et al., 2001). The sialic acid content in unfortified infant formulas is dependent on the sialic acids from bovine milk. As such, these formulas have lower total sialic acid content and different sialic acid proportions compared with human milk. To more closely match human milk composition, many manufacturers enrich infant formulas with sialic acidrich additives, such as whey protein (Lacomba et al., 2011). Therefore, robust methods are needed to determine sialic acids in dairy products and infant formulas. Sialic acid determination in a complex matrix, such as a dairy product, can be challenging. The majority of milk sialic acids are found as part of a glycoconjugate rather than as the free compound. In human milk, approximately $73 \%$ of sialic acid is bound to free oligosaccharides; however, previous infant formula research has shown that the commercial infant formulas contain sialic acids primarily bound to glycoproteins (Wang et al., 2001). Although spectroscopic methods exist that may quantify sialic acids bound to their glycoconjugates, these methods are prone to interference without prior sample purification and do not distinguish different sialic acids (Lacomba et al., 2010; Salcedo et al., 2011). To avoid interfering compounds and to quantify individual sialic acids, chromatographic methods are better suited to complex matrices such as infant formulas. To determine sialic acids by chromatographic methods, the sialic acids must first be released from the glycoproteins, glycolipids, and oligosaccharides. In 
dairy products, this is typically accomplished by using a dilute $(25-100 \mathrm{mM})$ sulfuric acid digestion at $80^{\circ} \mathrm{C}$ (Lacomba et al., 2010), but other acids that are volatile and can be removed by lyophilization have been evaluated, including acetic acid, trifluoroacetic acid, and $\mathrm{HCl}$ (Rohrer, 2000; Lacomba et al., 2010). Enzymatic methods using neuraminidases are available as well; however, they are limited to the cleavage specificity of the enzyme, which, depending on the glycoconjugates present in the sample, may lead to incomplete sialic acid release.

Following sample hydrolysis, 2 chromatographic methods are commonly used for sialic acid determinations. High-performance anion-exchange chromatography with pulsed amperometric detection (HPAEC-PAD), which is a direct determination method, or fluorescent labeling followed by UHPLC with fluorescence detection (UHPLC-FLD) have been used to determine sialic acids in a variety of dairy samples (Martín et al., 2007; Tang et al., 2008; Spichtig et al., 2010; Lacomba et al., 2011). Sialic acid fluorescent labeling using 1,2-diamino-4,5-methylenedioxybenzene dihydrochloride (DMB) was first reported for the evaluation of sialic acids in serum (Hara et al., 1986, 1989). This method has been modified to determine sialic acids in infant formulas (Martín et al., 2007; Spichtig et al., 2010; Lacomba et al., 2011). Although the fluorescent labeling method determines sialic acids indirectly, the chromatographic conditions are less likely to hydrolyze the $O$-acetylated sialic acids, allowing identification of a wider range of sialic acids (Klein et al., 1997). In this work, both HPAEC-PAD and UHPLC assays were compared after developing optimized sample preparations for each.

\section{MATERIALS AND METHODS}

\section{Reagents}

Deionized (DI) water, type I reagent grade, resistivity $\geq 18 \mathrm{M} \Omega$-cm (Milli-Q, Millipore, Billerica, MA) was used for all reagent preparations. Sodium hydroxide (50\%, wt/wt) was purchased from Fisher Scientific (Pittsburgh, PA). Sodium acetate, formic acid, sodium hydrosulfite, DMB, and 2-mercaptoethanol were purchased from Sigma (St. Louis, MO). Sulfuric acid and glacial acetic acid were from J.T. Baker (Phillipsburg, NJ). $\mathrm{N}$-Acetylneuraminic acid (Neu5Ac) and $N$ glycolylneuraminic acid (Neu5Gc) were purchased from Ferro Pfanstiehl (Waukegan, IL). Acetonitrile (HPLC grade) was purchased from Honeywell (Morristown, $\mathrm{NJ}$ ). A mixture of $\mathrm{O}$-acetylated sialic acids was purchased from Prozyme (San Leandro, CA).

\section{Samples}

Three commercially available infant formulas were analyzed. Brand A, an infant formula that did not have added maltodextrins (potentially interfering carbohydrates) in the ingredients list, but did list additional whey protein and galactooligosaccharides, was chosen as a typical infant formula. Brand B, an infant formula that contained added maltodextrins and rice starch, was chosen as a maltodextrin-rich sample. Brand C, a lactose-free, nondairy soy formula was chosen as a control sample that would not be expected to contain sialic acids.

\section{Release of Sialic Acids by Acid Hydrolysis}

Powdered infant formulas were prepared by a modified published method (Martín et al., 2007). Infant formula $(0.750 \mathrm{~g})$ was suspended in $10.0 \mathrm{~mL}$ of DI water. The samples were mixed using a vortexing mixer to ensure a homogeneous suspension. This mixture was hydrolyzed by adding $900 \mu \mathrm{L}$ of formula to $5.0 \mathrm{~mL}$ of $50 \mathrm{mM}$ sulfuric acid in a $7-\mathrm{mL}$ polypropylene screw-capped vial. Samples were also hydrolyzed by an alternate hydrolysis method by adding $2.5 \mathrm{~mL}$ of formula suspension to 2.5 $\mathrm{mL}$ of $100 \mathrm{~m} M$ sulfuric acid in a 7 -mL polypropylene, screw-capped vial with equivalent results. The capped vial was heated in a heat-block maintained at $80^{\circ} \mathrm{C}$ for $1 \mathrm{~h}$. After $1 \mathrm{~h}$, the samples were removed and allowed to cool to room temperature $\left(22^{\circ} \mathrm{C}\right)$ in approximately $10 \mathrm{~min}$. Before further treatment, the hydrolysates were centrifuged at $1.988 \times g$ and $5^{\circ} \mathrm{C}$ for $10 \mathrm{~min}$ to separate and remove the fats and proteins suspended in solution.

\section{HPAEC-PAD Sample Preparation}

After hydrolysis and centrifugation, samples were loaded on an anion-exchange resin (Thermo Scientific Dionex OnGuard II A cartridge, Thermo Scientific, Sunnyvale, CA) that was then washed with $10 \mathrm{~mL}$ of DI water to remove any residual neutral compounds from the resin. Sialic acids were then eluted with $25 \mathrm{~mL}$ of $50 \mathrm{mM} \mathrm{NaCl}$, filtered through a $0.2-\mu \mathrm{m}$ IC Acrodisc syringe filter (Pall Gelman, Port Washington, NY) and diluted 1:2.5 with DI water.

\section{UHPLC-FLD Sample Preparation}

Infant formulas were prepared, hydrolyzed, centrifuged, and loaded on an anion-exchange resin as described above. Samples were then eluted with $20 \mathrm{~mL}$ of $1 M$ formic acid, filtered through a $0.2-\mu \mathrm{m}$ IC syringe filter, and derivatized as described below. 


\section{Calibration Standards}

Calibration standards for $10-\mu \mathrm{L}$ injections for HPAEC-PAD sample analysis were prepared between 5.0 and $100 \mathrm{pmol}$ of Neu5Ac and between 0.34 and 6.8 pmol of Neu5Gc in DI water by diluting a standard stock solution of $0.10 \mathrm{~m} M$ Neu5Ac and $6.8 \mu M$ Neu $5 \mathrm{Gc}$ as needed. Standards were prepared daily from the stocks stored at $-40^{\circ} \mathrm{C}$.

Combined standards for UHPLC-FLD were prepared between 5 and $260 \mathrm{pmol}$ for Neu5Ac and between 0.2 and 9.8 pmol for Neu5Gc (based on a $5-\mu \mathrm{L}$ injection volume) in $1 M$ formic acid to match the matrix from the infant formula sample preparation.

\section{DMB Derivatization Conditions}

Samples and calibration standards were derivatized by adding $50 \mu \mathrm{L}$ of the DMB derivatization reagent (8 $\mathrm{m} M$ DMB, $1.5 M$ acetic acid, $14 \mathrm{~m} M$ sodium hydrosulfite, and $0.8 M 2$-mercaptoethanol) to $50 \mu \mathrm{L}$ of sample or standard in a $1.5-\mathrm{mL}$ screw-capped microcentrifuge vial. The solutions were incubated for $2.5 \mathrm{~h}$ in the dark at $50 \pm 2^{\circ} \mathrm{C}$. Samples, standards, and controls were derivatized at the same time with the same preparation of derivatization reagent. After $2.5 \mathrm{~h}$ of incubation, the solutions were cooled to quench the reaction. Derivatized samples may continue to slowly derivatize or degrade with exposure to light and oxygen and were analyzed as soon as possible to minimize the effect of continued reactions. The reagent is light sensitive and was stored at $-20^{\circ} \mathrm{C}$ in the dark when not in use. The stability of the DMB reagent solution is reported to be $1 \mathrm{yr}$ when stored in $200-\mu \mathrm{L}$ aliquots in amber microcentrifuge tubes at $-20^{\circ} \mathrm{C}$ (Reuter and Schauer, 1994; Spichtig et al., 2010); however, in this work, the derivatization reagent was prepared daily after observing discoloration of the initially colorless reagent upon overnight storage at $-20^{\circ} \mathrm{C}$.

\section{Chromatography-HPAEC-PAD}

For HPAEC-PAD, an ion chromatography system (Thermo Scientific Dionex ICS-3000) with a Thermo Scientific Dionex CarboPac PA20 column was used for separation and subsequent detection by pulsed amperometry via a disposable gold working electrode and a 4-potential waveform (Rocklin et al., 1998). Gradient conditions of 10 to $200 \mathrm{mM}$ sodium acetate in $100 \mathrm{mM}$ $\mathrm{NaOH}$ gradient from 0 to $15 \mathrm{~min}$ followed by $5 \mathrm{~min}$ at $200 \mathrm{~m} M$ sodium acetate in $100 \mathrm{~m} M \mathrm{NaOH}$ at a flow rate of $0.5 \mathrm{~mL} / \mathrm{min}$ were used to elute sialic acids. The column was equilibrated at $10 \mathrm{~m} M$ sodium acetate in $100 \mathrm{~m} M \mathrm{NaOH}$ for $5 \mathrm{~min}$ before injection of $10 \mu \mathrm{L}$ of sample. Both the column and detector were maintained at $30^{\circ} \mathrm{C}$.

\section{Chromatography-UHPLC-FLD}

The DMB-derivatized sialic acids were separated on a UHPLC system (Thermo Scientific Dionex Ultimate 3000 RSLC). Separation was performed on an Acclaim RSLC 120 C18 column $(2.2 \mu \mathrm{m}, 2.1 \times 100 \mathrm{~mm}$; Thermo Scientific) at a flow rate of $0.42 \mathrm{~mL} / \mathrm{min}$ and a temperature of $45^{\circ} \mathrm{C}$. A multi-step gradient separation beginning with an isocratic step of $5 \%$ acetonitrile for 5 min was used. This was followed by a linear gradient from 5 to $20 \%$ acetonitrile for $7 \mathrm{~min}$ and then a column wash step consisting of a 20 to $40 \%$ acetonitrile gradient in 2 min followed by $5 \mathrm{~min}$ of $40 \%$ acetonitrile. The column wash step removes strongly retained compounds that persist after sample preparation and DMB derivatization, and thereby preserves column performance. The column was equilibrated for $3 \mathrm{~min}$ at $5 \%$ acetonitrile before injecting $5 \mu \mathrm{L}$ of sample. Samples were maintained at $4^{\circ} \mathrm{C}$ in the dark in the autosampler. Analytes were detected by fluorescence with an excitation wavelength of $373 \mathrm{~nm}$ and emission wavelength of $448 \mathrm{~nm}$.

\section{RESULTS AND DISCUSSION}

Several sample preparation methods were investigated to remove potentially interfering compounds present in infant formulas. Because sialic acids are charged at neutral $\mathrm{pH}$, ion-exchange resins can be used to trap the sialic acids on the resin, while neutral compounds are not retained. The sialic acids can then be eluted with stronger eluent, such as formic acid, sodium acetate, or sodium chloride. If the eluent is volatile, it can be removed by lyophilization and the sample redissolved in DI water before analysis. However, lyophilization can be time consuming. To simplify sample preparation, multiple eluents were tested to evaluate resin loading and recovery of sialic acid standards from the anionexchange resin. Recovery was evaluated by making a direct injection on the column used for chromatography. The first eluent investigated was $1 M$ formic acid (Martín et al., 2007). Determination by HPAEC-PAD was not effective due to very low $(32-65 \%)$ recoveries, undoubtedly due to the high formic acid concentration. Other concentrations of formic acid, ranging from 100 to $250 \mathrm{mM}$, also showed poor recoveries of standards. Sodium acetate $(250 \mathrm{mM})$ was also investigated as a sample preparation eluent. Both incomplete elution from the sample preparation resin, as determined by sequential elution, and poor chromatography were observed with sodium acetate elution. Dilution of col- 
lected fractions from the sample preparation resin did not improve the chromatography.

Sodium chloride between 25 and $100 \mathrm{~m} M$ was evaluated as an eluent. Recoveries of both standards from the sample preparation resin and chromatography were good. Of the eluents tested, the best condition for HPAEC-PAD analysis was elution with $25 \mathrm{~mL}$ of $50 \mathrm{~m} M$ sodium chloride. This led to good recoveries (78-111\%) with minimal effect on the chromatography compared with standards prepared in DI water. However, these conditions could not be used for the UHPLC-FLD method, as will be discussed in the next section.

\section{Conditions Affecting Determination of DMB-Derivatized Sialic Acids}

In an early publication that reported the DMB method, Hara et al. (1989) noted that acid concentration affects derivatization yield. It is important that standards be derivatized in the same matrix as the sample to avoid systematic errors due to differing derivatization efficiencies. Table 1 illustrates the effect of acid concentration and sample matrix on the measured concentrations for Neu5Ac and Neu5Gc. For example, standards prepared in DI water that were derivatized by the DMB reagent described, which contains $750 \mathrm{mM}$ acetic acid, showed $57 \%$ of the peak area for Neu5Ac as the same concentration standards that were prepared in $1 M$ formic acid. After addition of the DMB derivatization reagent, these standards would be derivatized in a mixture of $750 \mathrm{~m} M$ acetic acid and $500 \mathrm{mM}$ formic acid. Furthermore, standards prepared in $50 \mathrm{mM}$ $\mathrm{NaCl}$ and derivatized showed peak area reductions for Neu5Ac and Neu5Gc compared with those prepared in both DI water and $1 M$ formic acid, which showed that the sample preparation method used for HPAEC-PAD could not be used with UHPLC-FLD.

Figure 1 illustrates the changes in peak height of the same concentration standard that results from changing the derivatization matrix. Samples containing high amounts of sodium chloride $(50 \mathrm{mM})$, such as that used to elute samples before HPAEC-PAD analysis, will degrade during the derivatization incubation time, leading to peak areas of $<35 \%$ of those without the added salt. Additional peaks, which were absent in blanks and samples derivatized without additional sodium chloride, were present in the retention window of sialic acids, between 4 and $12 \mathrm{~min}$, and also during the column wash. Standards in formic acid containing $5 \mathrm{mM}$ sodium chloride exhibited decreased peak areas of 12 to $13 \%$ compared with those without sodium chloride when prepared simultaneously with the same derivatization reagent. This effect was reduced compared with that at $50 \mathrm{~m} M$ sodium chloride and was similar to the between-day variability of $13 \%$ observed for the 67 pmol Neu5Ac standard across $4 \mathrm{~d}$ of derivatization. The efficiency of the derivatization reaction has a significant effect on the standard peak area from day to day. Preparing standards in parallel to samples limits the effect of this variability on analyte quantification; however, between-day peak areas were observed to vary by $13 \%$ for both Neu5Ac and Neu5Gc. Because of the observed changes in peak areas when derivatizing sialic acids with DMB in the presence of 50 $\mathrm{m} M$ sodium chloride, only acids were considered for use during sample preparation before derivatization. For the UHPLC-FLD analytical method, $1 M$ formic acid was used for the sample preparation steps.

For best accuracy, the standards must be derivatized in a matrix as similar as possible to the samples, including both acid and salt concentrations. Derivatization conditions for samples with high ionic strength should be optimized and should not be compared with standards prepared at lower ionic strength. Sialic acid release by neuraminidase is not appropriate for this determination method because the buffer used for sialic acid release is not acidic enough to allow efficient derivatization. This has been observed in our laboratory and by others (Harazono et al., 2011).

Table 1. Recovery of sialic acids based on the standard preparation matrix for 1,2-diamino-4,5methylenedioxybenzene dihydrochloride (DMB) derivatization

\begin{tabular}{llccc}
\hline & & \multicolumn{2}{c}{ Concentration $(\mu M)$} & \\
\cline { 3 - 4 } $\begin{array}{l}\text { Standard } \\
\text { matrix }\end{array}$ & Analyte $^{1}$ & Theoretical & Measured & $\begin{array}{c}\text { Recovery }^{2} \\
(\%)\end{array}$ \\
\hline \multirow{2}{*}{$\begin{array}{l}\text { M Formic acid } \\
50 \mathrm{~m} M \mathrm{NaCl}\end{array}$} & Neu5Ac & 3.7 & 5.2 & 140 \\
& Neu5Gc & 0.29 & 0.37 & 127 \\
Deionized water & Neu5Ac & 3.1 & 1.0 & 34 \\
& Neu5Gc & 0.24 & 0.087 & 36 \\
& Neu5Ac & 3.4 & 2.9 & 86 \\
& Neu5Gc & 0.26 & 0.23 & 89 \\
\hline
\end{tabular}

${ }^{1}$ Neu $5 \mathrm{Ac}=N$-acetylneuraminic acid; Neu5Gc $=N$-glycolylneuraminic acid.

${ }^{2}$ Recoveries are relative to a standard curve derivatized in $750 \mathrm{mM}$ acetic acid. 


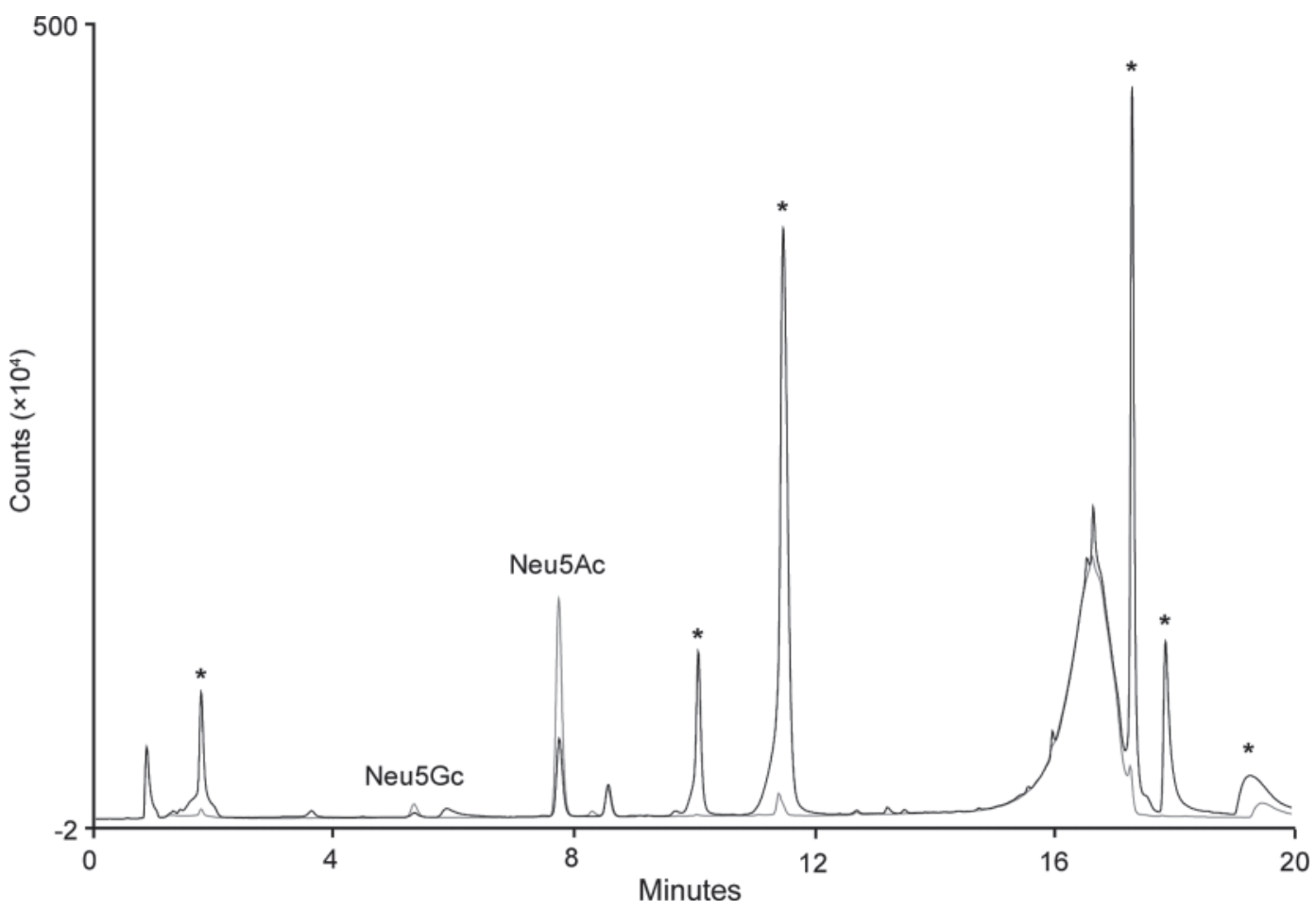

Figure 1. Response of standards derivatized with 1,2-diamino-4,5-methylenedioxybenzene dihydrochloride (DMB) in a total of $750 \mathrm{~m} M$ acetic acid (gray line) or $750 \mathrm{~m} M$ acetic acid with $50 \mathrm{mM} \mathrm{NaCl}$ (black line). Note the dramatic peak response reduction for $N$-acetylneuraminic acid (Neu5Ac) and $N$-glycolylneuraminic acid (Neu5Gc) standards derivatized in the presence of NaCl. Also note the additional peaks $(*)$ that are present in standards derivatized in the presence of $\mathrm{NaCl}$.

\section{Chromatographic Determination of Sialic Acids}

Linear Range, Limit of Quantification, Limit of Detection, and Precision. Table 2 shows the figures of merit for the HPAEC-PAD and UHPLC-FLD methods. In both cases, the response was linear for the range studied. Two calibration ranges for Neu5Ac were investigated, depending on the analytical method chosen, with $\leq 0.99$ correlation for UHPLC-FLD and 1.0 for HPAEC-PAD. The limit of detection (LOD) and limit of quantification (LOQ) were confirmed by standard injections that resulted in a response of 3 and 10 times the noise, respectively. When using HPAECPAD, Neu5Ac was determined to have an LOD of 0.24 pmol and an LOQ of 0.80 pmol. Similarly, Neu5Gc limits were found to be 0.21 and 0.70 pmol. By UHPLCFLD, the Neu5Ac LOQ and LOD were 0.17 and 0.06 pmol, and the Neu5Gc LOQ and LOD were 0.23 and 0.08 pmol, respectively.

Retention time and peak area precisions of standards were determined by 7 injections of a mid-range standard. Precision was equivalent by both assay methods,

Table 2. Comparison of linearity, limit of quantification (LOQ), limit of detection (LOD), and precision of sialic acid determination by high-performance anion-exchange chromatography with pulsed amperometric detection (HPAEC-PAD) and ultra-high-performance liquid chromatography with fluorescence detection (UHPLC-FLD) ${ }^{1}$

\begin{tabular}{lllccccc}
\hline Analyte & Method & $\begin{array}{c}\text { Range } \\
(\mathrm{pmol})\end{array}$ & $\mathrm{R}^{2}$ & $\begin{array}{c}\mathrm{LOQ}^{2} \\
(\mathrm{pmol})\end{array}$ & $\begin{array}{c}\mathrm{LOD}^{2} \\
(\mathrm{pmol})\end{array}$ & $\begin{array}{c}\text { RT } \\
(\mathrm{min})\end{array}$ & $\begin{array}{c}\text { Peak area } \\
\text { precision } \\
(\mathrm{RSD})\end{array}$ \\
\hline Neu5Ac & HPAEC-PAD & $5.0-100$ & 1.00 & 0.80 & 0.24 & 7.89 & 0.05 \\
& UHPLC-FLD & $5.0-260$ & 1.00 & 0.17 & 0.06 & 7.693 & 0.04 \\
Neu5Gc & HPAEC-PAD & $0.34-6.8$ & 1.00 & 0.70 & 0.21 & 13.86 & 0.05 \\
& UHPLC-FLD & $0.20-9.8$ & 0.99 & 0.23 & 0.08 & 5.286 & 0.55 \\
\hline
\end{tabular}

${ }^{1}$ Retention time (RT) and peak area precisions for HPAEC-PAD were measured by 7 injections of 25 pmol of $N$-acetylneuraminic acid (Neu5Ac) and $1.7 \mathrm{pmol}$ of $N$-glycolylneuraminic acid (Neu5Gc). Precisions for UHPLC-FLD were measured by 7 injections of a standard of 67 pmol of Neu5Ac and 5.2 pmol of Neu5Gc. RSD = relative standard deviation.

${ }^{2} \mathrm{LOD}$ and LOQ were confirmed by injections at the concentrations listed and measuring responses at 3 and 10 times the noise, respectively. 
with retention time relative standard deviations (RSD) of 0.03 to 0.05 and peak area RSD of 0.50 to 1.98 .

Determination of Sialic Acids in Infant Formulas. Sialic acid separation on a C18 column with an acetonitrile gradient by UHPLC-FLD is shown in Figure 2: Neu5Gc eluted first, followed by Neu5Ac, $\mathrm{N}$-acetyl-7-O-acetylneuraminic acid (Neu5,7Ac2), $\mathrm{N}$ glycolyl-9-O-acetylneuraminic acid (Neu5Gc9Ac), $N$ acetyl-9-O-acetylneuraminic acid (Neu5,9Ac2), and $\mathrm{N}$ acetyl-6 (8) 9-O-diacetylneuraminic acid (Neu5,7(8) 9 Ac3). The 2 primary sialic acids of interest, Neu5Ac and Neu5Gc, were well resolved. As expected, the dominant sialic acid present in dairy-based formulas was Neu5Ac (peak 2), whereas Neu5Gc (peak 1) was present to a lesser extent. Brand A also contained minor amounts of Neu5,7Ac2, and brands A and B contained a small amount of Neu5,9Ac2. The strong hydrolysis conditions used to release sialic acids in these samples were not optimal for determining these sialic acids; however, they are present. As expected, brand C, a soy-based formula, did not contain the identified sialic acids. However, it should be noted that, under the gradient conditions described here, a small, unknown peak was observed that elutes near Neu5Gc and could potentially interfere with determination of this sialic acid. Different acetonitrile gradient elutions and use of an isocratic method consisting of 8:7:85 methanol:acetonitrile:water did not fully resolve this peak from Neu5Gc (data not shown). Previously published work by Martín et al. (2007) and Spichtig et al. (2010) did not show this peak under isocratic conditions and it is likely dependent on the formulation of soy infant formula. However, the retention time was 0.36 min shorter than that of Neu5Gc and there was evidence of 2 components eluting in spiked samples. For a single day of triplicate sample analysis, an average of $94 \pm 4.9 \mathrm{mg}$ of Neu5Ac per 100 $\mathrm{g}$ of infant formula was determined in brand $\mathrm{A}$ and 56 $\pm 6.8 \mathrm{mg}$ in brand $\mathrm{B}$ (Table 3 ).

Figure 3 shows the separation of the same 3 infant formulas by HPAEC-PAD as well as a soy infant formula spiked with Neu5Ac and Neu5Gc. N-Acetylneuraminic acid eluted early and was well resolved from interfering peaks under these conditions, including peaks from other carbohydrates that were minimized by the sample preparation discussed earlier. This is most easily seen in the chromatography of a sialic acid-spiked soy for-

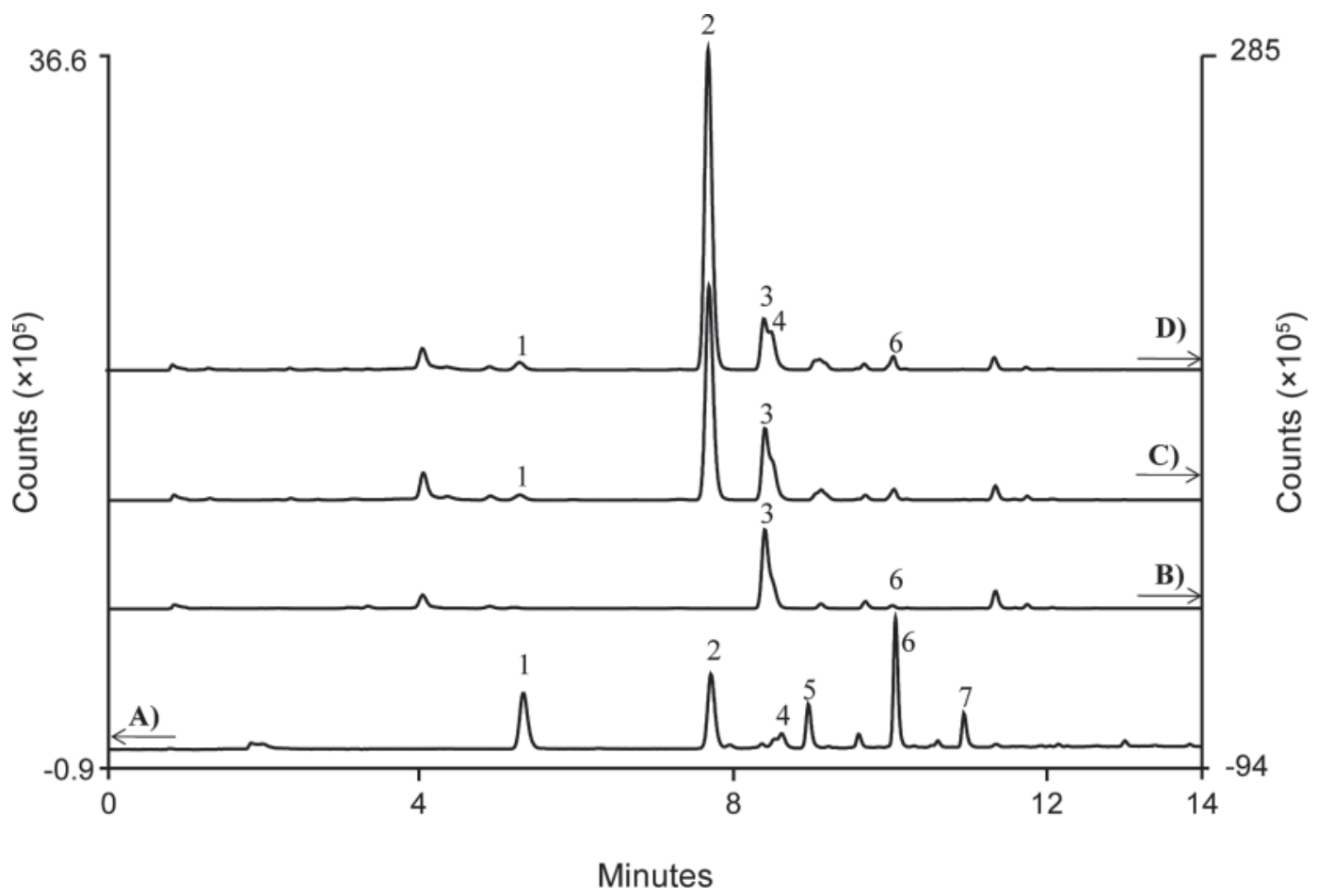

Figure 2. Ultra-high-performance liquid chromatography with fluorescence detection (UHPLC-FLD) determination of sialic acids after 1,2-diamino-4,5-methylenedioxybenzene dihydrochloride (DMB) derivatization with 18\% signal offset. (A) Combined sialic acid standards; (B) to (D) formula samples based on (B) soy with added maltodextrins, (C) dairy with added maltodextrins [49 pmol of $N$-acetylneuraminic acid (Neu5Ac), $1.4 \mathrm{pmol}$ of $\mathrm{N}$-glycolylneuraminic acid (Neu5Gc)], and (D) dairy without added maltodextrins (72 pmol of Neu5Ac, 1.9 pmol of Neu5Gc). Peak $1=$ Neu5Gc, peak $2=$ Neu5Ac, peak $3=$ DMB reagent, peak $4=N$-acetyl-7-O-acetylneuraminic acid $($ Neu5,7Ac2), peak $5=$ $\mathrm{N}$-glycolyl-9-O-acetylneuraminic acid (Neu5Gc9Ac), peak $6=\mathrm{N}$-acetyl-9-O-acetylneuraminic acid (Neu5,9Ac2), peak $7=\mathrm{N}$-acetyl-7,(8),9-Oacetylneuraminic acid (Neu5,7(8),9Ac3). Note that chromatogram A is on an expanded scale. 
Table 3. Results of triplicate sample analysis of 2 brands of infant formula by ultra-high-performance liquid chromatography with fluorescence detection (UHPLC-FLD)

\begin{tabular}{|c|c|c|c|c|c|c|c|}
\hline Sample & Analyte $^{1}$ & $\begin{array}{l}\text { Determined } \\
\text { amount } \\
(\mathrm{pmol})\end{array}$ & $\begin{array}{c}\text { mg of analyte/100 } \\
\mathrm{g} \\
\text { of sample }\end{array}$ & $\begin{array}{l}\text { Peak area } \\
\text { precision } \\
\left(\mathrm{RSD}^{2}\right)\end{array}$ & $\begin{array}{l}\text { Retention time } \\
\text { precision } \\
(\mathrm{RSD})\end{array}$ & $\begin{array}{l}\text { Sample analysis } \\
\text { precision } \\
(\mathrm{RSD})\end{array}$ & $\begin{array}{l}\text { Mean } \pm \mathrm{SD} \\
\quad(\mathrm{mg} \text { of } \\
\text { analyte } / 100 \\
\mathrm{~g} \text { of sample) }\end{array}$ \\
\hline \multicolumn{8}{|l|}{ Brand A } \\
\hline \multirow[t]{2}{*}{ Replicate 1} & Neu5Ac & 70.2 & 91 & 3.95 & 0.05 & 5.2 & $94 \pm 4.9$ \\
\hline & Neu5Gc & 1.86 & 2.5 & 3.99 & 0.07 & 7.8 & $2.7 \pm 0.21$ \\
\hline \multirow[t]{2}{*}{ Replicate 2} & Neu5Ac & 70.4 & 92 & 0.40 & 0.05 & & \\
\hline & Neu 5 Gc & 1.90 & 2.6 & 1.16 & 0.09 & & \\
\hline \multirow[t]{2}{*}{ Replicate 3} & Neu $5 \mathrm{Ac}$ & 80.1 & 100 & 0.28 & 0.05 & & \\
\hline & Neu 5 Gc & 2.14 & 2.9 & 1.67 & 0.06 & & \\
\hline \multicolumn{8}{|l|}{ Brand B } \\
\hline \multirow[t]{2}{*}{ Replicate 1} & Neu $5 \mathrm{Ac}$ & 38.7 & 50 & 1.59 & 0.08 & 12 & $56 \pm 6.5$ \\
\hline & Neu5Gc & 1.06 & 1.4 & 1.97 & 0.14 & 15 & $1.5 \pm 0.23$ \\
\hline \multirow[t]{2}{*}{ Replicate 2} & Neu 5 Ac & 49.1 & 63 & 1.47 & 0.03 & & \\
\hline & Neu5Gc & 1.36 & 1.8 & 1.85 & 0.02 & & \\
\hline \multirow[t]{2}{*}{ Replicate 3} & Neu5Ac & 42.4 & 56 & 1.84 & 0.05 & & \\
\hline & Neu $5 \mathrm{Gc}$ & 1.03 & 1.4 & 2.25 & 0.06 & & \\
\hline
\end{tabular}

${ }^{1}$ Neu5Ac $=N$-acetylneuraminic acid; Neu5Gc $=N$-glycolylneuraminic acid.

${ }^{2}$ Relative standard deviation.

mula sample (chromatogram B) where the other carbohydrates in the formula persist. $N$-Acetylneuraminic acid (peak 2) was well resolved from neighboring peaks and Neu5Gc (peak 3) was also separated from a nearby potential interference. More rapid gradients were investigated to reduce the time required for separation, but were found to be inappropriate for these samples due to these potentially interfering peaks. Notable is the presence of residual lactose in dairy-based formulas and likely maltodextrins in brands $\mathrm{B}$ and $\mathrm{C}$ that specifically listed maltodextran as an ingredient. For a single day of triplicate analysis, the prepared samples of brands A and B contained $86 \pm 0.5$ and $47 \pm 7.4 \mathrm{mg}$ of Neu5Ac in $100 \mathrm{~g}$ of sample, respectively (Table 4).

Precision and Accuracy of Sample Analysis. Samples were analyzed in triplicate to evaluate the precision of the assay by UHPLC-FLD after DMB derivatization. Table 3 details the results for a single day of analysis. Peak area RSD for Neu5Ac were generally $<2$. Retention times were stable with RSD $<0.2$, indicating that under these gradient conditions, neutral sample components elute from the column and do not affect subsequent analyses. The assay precision (RSD) determined with triplicate samples was 7.7 for

Table 4. Results of triplicate sample analysis of 2 brands of infant formula by high-performance anion-exchange chromatography with pulsed amperometric detection (HPAEC-PAD)

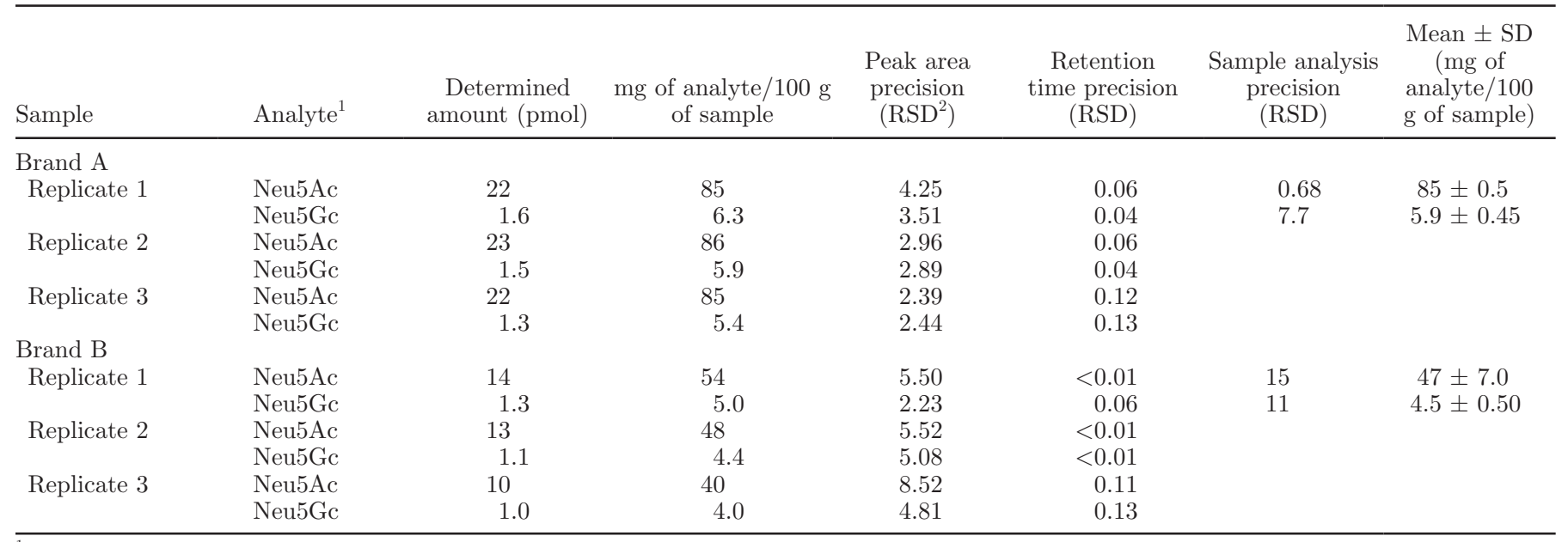

${ }^{1}$ Neu5Ac $=N$-acetylneuraminic acid; Neu5Gc $=N$-glycolylneuraminic acid.

${ }^{2}$ Relative standard deviation. 
Neu5Ac and 8.0 for Neu5Gc for brand A. Replicates of brand $\mathrm{B}$ were more variable, with RSD of 12 and 16 for Neu5Ac and Neu5Gc, respectively. Sample C, a soy-based formula, did not contain measurable sialic acids, which was expected for an unfortified, nondairy infant formula.

When analyzing the same 3 infant formula samples by HPAEC-PAD (Table 4), the results were similar to those obtained using the UHPLC-FLD method. Sample $\mathrm{C}$, the soy-based infant formula, did not contain detectable Neu5Ac or Neu5Gc and was used as a blank matrix for comparing recovery of spiked sialic acids. Retention time precision was similar to that determined by injecting standards, with RSD ranging from $<0.01$ to 0.13 . Peak area precision RSD ranged from 2.23 to 8.52.

Variability within sample replicates can be large, as illustrated in Tables 3 and 4 , and therefore optimization of the digestion and sample preparation methods for individual infant formulas is required. By both chromatographic methods, brand $\mathrm{B}$ results were more variable than brand A, suggesting that the sample matrix affected assay precision for brand B..
Between-day precision was evaluated by repeating sample preparation and analysis on multiple days. When comparing the average determined amounts, betweenday precision (RSD) was 1.3 and 1.0 for Neu5Ac and 6.6 and 8.9 for Neu5Gc in brands A and B, respectively, for the UHPLC-FLD assay. Between-day precision of HPAEC-PAD assay ranged from 8.9 (brand B) to 11 (brand A) for Neu5Gc and from 11 (brand B) to 18 (brand A) for Neu5Ac over $3 \mathrm{~d}$ of sample assay (data not shown).

The determined values obtained by DMB derivatization appeared to be systematically higher than those determined directly by HPAEC-PAD. However, the variability in the sample replicates was of the same scale as the differences between the methods. To further investigate performance of both methods, accuracy was evaluated by spiking the sample hydrolysates with known amounts of Neu5Ac and Neu5Gc after acid hydrolysis but before sample preparation by anion exchange. This spiking was also done in a hydrolysis reagent blank and soy formula samples for comparison. For samples that did not contain sialic acids, 75 pmol

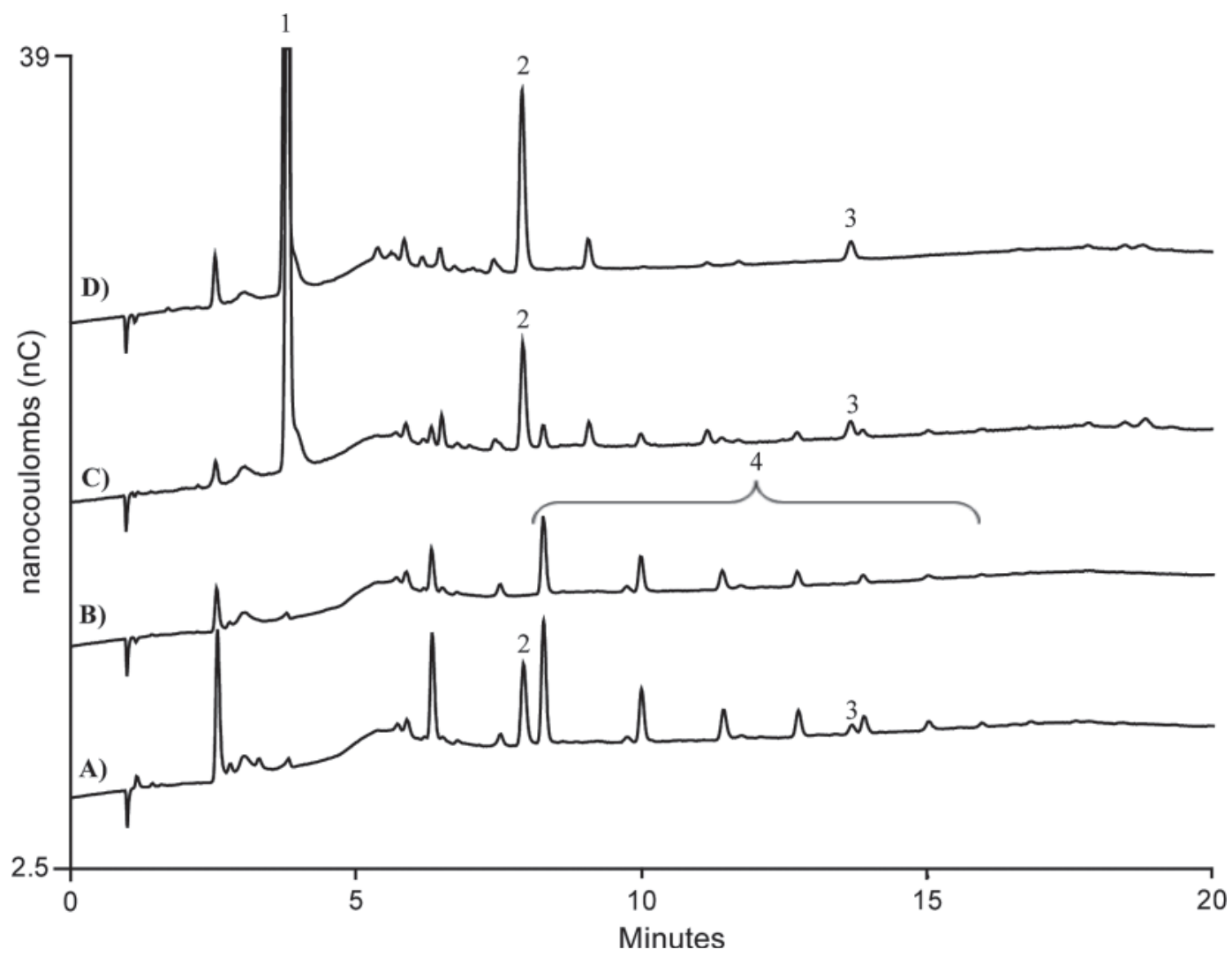

Figure 3. High-performance anion-exchange chromatography with pulsed amperometric detection (HPAEC-PAD) determination of infant formula samples with $21 \%$ signal offset: (A) soy-based formula spiked with $N$-acetylneuraminic acid (Neu5Ac; 7.5 pmol) and $N$-glycolylneuraminic acid (Neu5Gc; $0.48 \mathrm{pmol}$ ); (B) soy-based formula with added maltodextrins; (C) dairy-based formula with added maltodextrins (11 pmol of Neu5Ac, 1.2 pmol of Neu5Gc); and (D) dairy-based without added maltodextrins (19 pmol of Neu5Ac, 1.5 pmol of Neu5Gc). Peak $1=$ lactose, peak $2=$ Neu5Ac, peak $3=$ Neu5Gc, peak $4=$ maltodextrins. 
Table 5. Sialic acid recovery in 3 infant formulas using high-performance anion-exchange chromatography with pulsed amperometric detection (HPAEC-PAD) and ultra-high-performance liquid chromatography with fluorescence detection (UHPLC-FLD)

\begin{tabular}{llcc}
\hline & & \multicolumn{2}{c}{ Recovery (\%) } \\
\cline { 3 - 4 } Sample & Analyte $^{1}$ & HPAEC-PAD & UHPLC-FLD \\
\hline Brand A & Neu5Ac & 108 & 100 \\
& Neu5Gc & 111 & 120 \\
Brand B & Neu5Ac & 100 & 120 \\
& Neu5Gc & 95 & 120 \\
Brand C & Neu5Ac & 96 & 95 \\
& Neu5Gc & 100 & 95 \\
Hydrolysis blank & Neu5Ac & 80 & 92 \\
& Neu5Gc & 78 & 89
\end{tabular}

${ }^{1}$ Neu5Ac $=N$-acetylneuraminic acid; Neu5Gc $=N$-glycolylneuraminic acid.

of Neu5Ac and 5.8 pmol of Neu5Gc were added. Brands $\mathrm{A}$ and $\mathrm{B}$ were spiked with amounts equal to the previously determined amounts. Recoveries from samples measured by UHPLC-FLD ranged from 89 to $120 \%$ (Table 5). Recoveries were higher in dairy-based infant formulas compared with the soy infant formula and reagent blank samples used as controls. Accuracy can be highly affected by the efficiency of the derivatization, which, as noted earlier, can be affected by the matrix of the derivatization reaction. When repeating the same experiment by HPAEC-PAD, recoveries for Neu5Ac ranged from 80 to $108 \%$ for the 3 infant formulas and recoveries for Neu5Gc were similar, ranging from 78 to $111 \%$.

\section{Comparison of Sample Preparation for the Methods}

High-performance anion-exchange chromatography with pulsed amperometric detection is a direct method that does not require derivatization; however, the alkaline elution conditions deacetylate the oxygen-linked acetyl groups of any $O$-acetylated sialic acids that survive acid hydrolysis, converting them to Neu5Ac and Neu5Gc and preventing their direct determination. If only Neu5Ac and Neu5Gc are of interest, either method is appropriate. The time required for sample analysis by the 2 methods is different. Both methods require the same sample hydrolysis optimization and anionexchange sample preparation for consistent sample analysis. These steps take approximately $4 \mathrm{~h}$, in total, for a set of 3 triplicate samples and controls (12 digestions). In addition to the sample preparation time, derivatization will require $2.5 \mathrm{~h}$ for the reaction with an additional $1 \mathrm{~h}$ of time to stop the reaction and prepare the samples for injection after the derivatization is complete.

\section{CONCLUSIONS}

In this work, HPAEC-PAD and fluorescent labeling followed by UHPLC-FLD methods were compared for determination of $\mathrm{N}$-acetylated sialic acids in infant formulas. Two methods of sample preparation were developed, one to simplify sample preparation before HPAEC-PAD and a second to optimize DMB derivatization before UHPLC-FLD. These methods are not interchangeable because of the changes in DMB reaction efficiency in the presence of sodium chloride as well as low recovery from formic acid sample preparation when using HPAEC-PAD. Assay results by both chromatographic methods showed a linear response and sufficient sensitivity to analyze the samples, with some sensitivity advantage when using fluorescence detection. Assay precision was similar by both methods, with both assays showing a precision dependence on the infant formula analyzed, based on the analysis of 3 distinct samples with different formulations. Assay accuracy was good by both methods, provided that the standards were DMB derivatized in a matrix that matched the samples for the UHPLC-FLD method. Differences in the derivatization conditions between standards and samples can change the reaction efficiency and strongly affect quantification. Overall, HPAEC-PAD has faster sample preparation, because it directly detects the sialic acids, removing the need for derivatization. For samples that are difficult to matrix match or have a matrix that leads to poor DMB derivatization, HPAEC-PAD is preferred. For samples in which quantification of $O$-acetylated sialic acids is needed or for samples with low concentrations of sialic acids, derivatization followed by UHPLC with fluorescence detection is preferred.

\section{REFERENCES}

Hara, S., M. Yamaguchi, Y. Takemori, K. Furuhata, H. Ogura, and M. Nakamura. 1989. Determination of mono- $O$-acetylated $\mathrm{N}$-acetylneuraminic acids in human and rat sera by fluorometric high-performance liquid chromatography. Anal. Biochem. 179:162-166.

Hara, S., M. Yamaguchi, Y. Takemori, and M. Nakamura. 1986. Highly sensitive determination of $\mathrm{N}$-acetyl- and $\mathrm{N}$-glycolylneuraminic acids in human serum and urine and rat serum by reversed-phase liquid chromatography with fluorescence detection. J. Chromatogr. 377:111-119.

Harazono, A., T. Kobayashi, N. Kawasaki, S. Itoh, M. Tada, N. Hashii, A. Ishii, T. Arato, S. Yanagihara, Y. Yagi, A. Koga, Y. Tsuda, M. Kimura, M. Sakita, S. Kitamura, H. Yamaguchi, H. Mimura, Y. Murata, Y. Hamazume, T. Sato, S. Natsuka, K. Kakehi, M. Kinoshita, S. Watanabe, and T. Yamaguchi. 2011. A comparative study of monosaccharide composition analysis as a carbohydrate test for biopharmaceuticals. Biologicals 39:171-180.

Klein, A., S. Diaz, I. Ferreira, G. Lamblin, P. Roussel, and A. E. Manzi. 1997. New sialic acids from biological sources identified by a comprehensive and sensitive approach: Liquid chromatography- 
electrospray ionization-mass spectrometry (LC-ESI-MS) of SIA quinoxalinones. Glycobiology 7:421-432.

Lacomba, R., J. Salcedo, A. Alegría, R. Barberá, P. Hueso, E. Matencio, and M. J. Lagarda. 2011. Sialic acid ( $N$-acetyl and $N$-glycolylneuraminic acid) and ganglioside in whey protein concentrates and infant formulae. Int. Dairy J. 21:887-895.

Lacomba, R., J. Salcedo, A. Alegría, M. J. Lagarda, R. Barberá, and E. Mantencio. 2010. Determination of sialic acid and gangliosides in biological samples and dairy products. J. Pharm. Biomed. Anal. 51:346-357.

Martín, M. J., E. Vázquez, and R. Rueda. 2007. Application of a sensitive fluorometric HPLC assay to determine the sialic acid content of infant formulas. Anal. Bioanal. Chem. 387:2943-2949.

Reuter, G., and R. Schauer. 1994. Determination of sialic acids. Methods Enzymol. 230:168-199.

Rocklin, R. D., A. P. Clarke, and M. Weitzhandler. 1998. Improved long-term reproducibility for pulsed amperometric detection of carbohydrates via a new quadruple-potential waveform. Anal. Chem. 70:1496-1501.
Rohrer, J. S. 2000. Analyzing sialic acids using high-performance anion-exchange chromatography with pulsed amperometric detection. Anal. Biochem. 283:3-9.

Salcedo, J., R. Lacomba, A. Alegria, R. Barbera, E. Matencio, and M. J. Lagarda. 2011. Comparison of spectrophotometric and HPLC methods for determining sialic acid in infant formulas. Food Chem. 127:1905-1910.

Spichtig, V., J. Michaud, and S. Austin. 2010. Determination of sialic acids in milks and milk-based products. Anal. Biochem. 405:28 40.

Tang, K.-T., L.-N. Liang, Y.-Q. Cai, and S.-F. Mou. 2008. Determination of sialic acid in milk and products using high performance anion-exchange chromatography coupled with pulsed amperometric detection. Chin. J. Anal. Chem. 36:1535-1538.

Wang, B., and J. Brand-Miller. 2003. The role and potential of sialic acid in human nutrition. Eur. J. Clin. Nutr. 57:1351-1369.

Wang, B., J. Brand-Miller, P. McVeigh, and P. Petocz. 2001. Concentration and distribution of sialic acid in human milk and infant formulas. Am. J. Clin. Nutr. 74:510-515. 